\title{
Replanteamiento de la Estrategia 100x100 a partir de un Índice de Desarrollo Humano para Localidades
}

\section{Rethinking Estrategia 100x100 using a Human Development Index for Localities}

\author{
Francisco José Zamudio-SÁnchez* \\ Roxana Ivette Arana-Ovalle* \\ Javier Santibáñez-Cortés*
}

\begin{abstract}
In this paper we present an index to measure human development. We take as basis the principles by Amartya Sen, and as an operative way, a statistical metric. We compare the results of selecting a set of municipalities using our index, against the ones included in the so called "Estrategia 100x100". The selection indicates that the strategy should be modified and that the proposed index relates better with other social indexes currently used. Our methodology is quite general to build an index, so it can be used to obtain measures of other social features.
\end{abstract}

Keywords: public policy, social measurement, human development, quality of life

\section{Resumen}

En este artículo se presenta un índice para medir el desarrollo humano. Se toman como bases conceptuales los principios de Amartya Sen y, como forma operativa, una métrica estadística. Comparamos los resultados de un conjunto de municipios con el índice propuesto, contra los municipios incluidos en la denominada Estrategia 100x100. La selección indica que la estrategia debe ser modificada y que el índice propuesto se relaciona mejor con otros índices sociales actualmente usados. Nuestra metodología es muy general para construir un índice y puede ser usada para obtener medidas de otras características sociales.

Palabras clave: política pública, medición social, desarrollo humano, calidad de vida.

*Universidad Autónoma Chapingo, México. Correos-e: fzamudios@taurus.chapingo.mx, aovalle@ colpos.mx y jsantibanezc@yahoo.com 


\section{Introducción}

La Secretaría de Desarrollo Social (Sedesol) es el organismo gubernamental encargado de la coordinación de los programas sociales. En el marco de operación de las estrategias que aplica se especifica la población, objetivo de las intervenciones; en algunos casos, se usan métricas sociales para apoyar los criterios de selección de los beneficiarios. Actualmente este organismo desarrolla, entre otros, el Programa de Inclusión Social "Próspera”, en el que se utilizó el Índice de Marginación (IM) del Consejo Nacional de Población (Conapo) y el Índice de Rezago Social (IRS) del Consejo Nacional de Evaluación de Política Pública (Coneval), para seleccionar localidades factibles de ser apoyadas. Además, estos dos organismos (Sedesol y Coneval) eligen a las familias beneficiarias en las localidades previamente seleccionadas con base en las líneas de bienestar mínimo. Así también, el Programa de Apoyo Alimentario es otro ejemplo de uso de medidas sociales que emplea las líneas de bienestar para la selección de familias beneficiarias.

En México hay más programas sociales con el objetivo de mejorar las condiciones de vida y el bienestar de la población, por ejemplo, la atención a jornaleros agrícolas, el desarrollo de zonas prioritarias, estancias infantiles, pensión para adultos mayores, entre otros. Los beneficiarios de algunos de ellos están determinados de manera precisa; sin embargo, existen casos, como el del desarrollo de zonas prioritarias, en el que se plantea ayudar a las regiones más necesitadas, a partir de un marcado uso de índices e indicadores para determinar a los posibles beneficiarios; no obstante, es relevante analizar y debatir sobre los criterios con los que se identifican a los más necesitados.

Para caracterizar a la población con más carencias, se consideran múltiples aspectos: nivel de ingresos, acceso a la salud y educación, participación política, cohesión social, entre otros. Para el propósito de este trabajo mencionaremos tres conceptos que reúnen, de alguna forma, las diferentes caras de la vulnerabilidad presente en la población mexicana: el rezago social, la pobreza y el desarrollo humano $(\mathrm{DH})$.

El Coneval propone una medida para calcular el índice de rezago social (IRS), y aunque no emite un concepto sobre este término, esta clasificación concentra información sobre carencias en educación, salud, servicios básicos y espacios en la vivienda. Los datos que agrupa se basan en los criterios establecidos en la Ley General de Desarrollo Social y se evalúan a escala local, estatal y nacional; la ventaja de este indicador es que permite realizar un ordenamiento según el nivel de rezago. Los detalles técnicos sobre las variables del IRS se pueden consultar en las publicaciones del Coneval (2007). La información más actual que se tiene de 
este índice es de 2010, en donde se advierte que Chiapas, Guerrero y Oaxaca fueron las entidades con mayor rezago social, mientras que Coahuila, el Distrito Federal y Nuevo León fueron las de menor rezago.

El Comité Técnico de Medición de la Pobreza en 2002 propuso que la pobreza se mide a partir de rubros que representan los costos por adquirir determinadas canastas de bienes y servicios. De los tres indicadores de pobreza considerados, sólo el primero de ellos es relevante para este trabajo, ya que permite identificar a los hogares en situación de pobreza alimentaria, lo que implica que no existe la capacidad de cubrir sus necesidades básicas de alimentación (Coneval, 2013a). En agosto del 2000 se estableció que un hogar en situación de pobreza alimentaria es aquel con un ingreso diario por integrante de 15,4 o 20,9 pesos, ya sea que se encuentre ubicado en un área rural o urbana (Cortés, 2003). Los resultados vigentes de esta medida hacen referencia a 2010 y en ellos se indica que $18,8 \%$ de la población (21,2 millones de personas) se encontraba en condiciones de pobreza alimentaria (Coneval, 2011a).

El desarrollo humano se define como "el proceso que ofrece a las personas incrementar las oportunidades, entre otras, a una vida prolongada y saludable, acceso a la educación y disfrutar de un nivel de vida decente" (PNUD, 1990: 33). Este concepto se presentó en el Primer Informe de Desarrollo Humano del Programa de las Naciones Unidas para el Desarrollo (PNUD). La medida del DH es el Índice de Desarrollo Humano (IDH) y se calcula como la media aritmética (actualmente como la media geométrica) de los tres indicadores básicos del DH: salud, educación e ingresos. El IDH es posiblemente uno de los índices más conocidos para medir el bienestar (Osberg y Sharpe, 2005), principalmente después de que se generalizó la idea de que el desarrollo va más allá de lo económico.

Las cifras más recientes del IDH de los municipios de México fueron presentadas en 2014 por el PNUD, con información de 2010 (PNUD, 2014). Utilizamos estos resultados para validar las mediciones que se obtienen con el índice que proponemos pero, en cuanto a la metodología de cálculo del IDH, nos concentraremos en la que se basó la Conapo en 2001, para evaluar el DH en los municipios con información del 2000, porque fueron estos resultados los utilizados para seleccionar a los municipios de la Estrategia 100x100.

La Sedesol -mediante la reorientación y focalización de diversas políticas públicas y con el propósito de mejorar las condiciones de rezago de los municipios más desfavorecidos, identificados principalmente a través del IDH- en 2007 puso en marcha la Estrategia 100x100 (E100x100), la cual tiene seis ejes rectores: educación, salud, generación de opciones de ingreso y fortalecimiento de la actividad económica, infraestructura 
social básica, vivienda y protección de los recursos naturales; en total contemplan 41 acciones principales para contribuir al desarrollo social. Sus objetivos son aumentar el ingreso y la calidad de vida de los habitantes de los municipios más vulnerables, mediante la creación de empleos y el mejoramiento del acceso a servicios básicos como la salud y la educación (Sedesol, 2010).

El campo de acción de la E100x100 está conformado por los 125 municipios con menor IDH. De manera específica, la selección de los 125 municipios de la E100x100 se realizó con base en los siguientes criterios:

i) 100 municipios con menor IDH en 2000 .

ii) Aquellos municipios con nivel de rezago social muy alto y con más de $60 \%$ de su población en condiciones de pobreza alimentaria.

Sobre la pertinencia de las medidas empleadas (el IRS, la línea de pobreza alimentaria y el IDH), argumentamos que el IDH no es una medida que responda a la dinámica interna del país, caracterizada por la presencia de múltiples asentamientos humanos de tamaño demográfico reducido y dispersos por todo el territorio, a diferencia de los otros dos indicadores, que son construidos exprofeso para ser sensibles a la problemática social mexicana. Además, debido a que la información con la que fue construida la selección data del 2000 , consideramos que la información acerca de la situación de los 125 municipios no está actualizada y que este problema de vigencia puede ocasionar su no legitimidad actualmente.

Por tanto, más allá de evaluar el impacto de la E100x100, nos concentraremos en determinar la pertinencia de las mediciones empleadas y la vigencia de la información que se emplea en la selección de los municipios. En esta investigación se pretende proponer una medida propia a las condiciones de DH del país, para determinar de manera específica si después de 10 años las condiciones de los municipios en el universo de la estrategia aún son desfavorables, y en caso afirmativo, si se trata de los 125 en las condiciones más adversas. Debe notarse en lo que sigue que la medida se construye para el DH, no obstante puede ser usada para construir prácticamente cualquier medida social.

En este trabajo presentamos un Índice de Desarrollo Humano en las Localidades (IDHL), que también puede ser evaluado a escala municipal, estatal y nacional. El índice que proponemos se basa en las ideas de Sen sobre la conceptualización del DH (PNUD, 1990). El IDHL permite inducir un ordenamiento consistente que facilita la identificación de la población más vulnerable de los municipios de México, lo que podría permitir una mejor planeación de los programas sociales. 
El enfoque que presentamos considera atributos que están directamente relacionados con la característica a medir, en este caso, con las opciones para el desarrollo de las capacidades. La contribución de nuestro trabajo consiste en concebir el cálculo de los índices como una distancia estadística, basada en las varianzas y covarianzas de las variables involucradas, y no como una combinación lineal (promedio ponderado). Esto tiene como implicaciones que:

i) Los índices que resultan son sensibles a cambios en los valores de los atributos, ya que éstos son ponderados de acuerdo a su variabilidad.

ii) Los índices consideran las diversas relaciones que existen entre los atributos, para hacer una medición más apegada a la realidad, en el sentido que se toma en cuenta la influencia que estas relaciones tienen sobre la característica a medir.

La contribución más relevante del trabajo en cuanto a la construcción del IDHL es la propiedad descrita en el inciso ii), ya que en general las mediciones del DH no consideran las interacciones entre los diversos atributos que componen la medida, y con ello se pierde la información específica asociada al territorio bajo análisis.

Nuestra propuesta sugiere una nueva forma para construir índices sociales (aunque aquí se ejemplifica con el IDHL), cuyo uso es frecuente en la planeación de las políticas respectivas. Está basado en una métrica estadística cuyas propiedades pueden consultarse en Johnson y Wichern (1999). Además, la correspondencia entre la forma final del índice y la que indica la teoría del DH las variables deben contribuir a éste, pero se yuxtaponen. El que sea una métrica nos garantiza poder ordenar a los territorios y con ello seleccionar aquellos que están en las condiciones más desfavorables y que la forma del índice refleje lo indicado en la teoría del $\mathrm{DH}$, asegura que la medición sea de las opciones con las que los habitantes cuentan para desarrollar sus capacidades. Nuestra propuesta metodológica posibilita construir índices que simultáneamente ordenen, consideren la variación de los atributos estudiados e incorporen las diversas interacciones que se dan entre los mismos.

\section{Metodología}

Para introducir una nueva metodología en la elaboración de índices sociales, utilizamos como ejemplo el DH de los municipios de México. El resultado es el índice al que hemos denominado IDHL. Con los resultados del IDHL para evaluar el DH en los municipios del país, realizamos 
una selección de los 125 con las medidas más bajas, es decir, los que presentan las mayores deficiencias en las características consideradas. Comparamos esta selección de municipios con los que conformaron la E100x100, la cual seleccionamos por ser un claro ejemplo del uso de indicadores en la planeación de programas sociales. Para responder a los cuestionamientos planteados en la introducción, realizamos una comparación entre las situaciones en 2010 de los municipios considerados en la E100x100 y 125 municipios que identificamos como los más vulnerables de acuerdo con ciertos criterios, análogos a los usados en 2007.

Esta comparación ayudó a determinar que algunos de los municipios contemplados en la E100x100 deberían seguir considerándose prioritarios, mientras que el resto, al haber mejorado su situación, deberían dejar de ser parte de la estrategia. Además, pudimos validar que la metodología que proponemos es más sensible en cuanto a que permite detectar variaciones más pequeñas en las características de la población.

Las fuentes de información utilizadas son los Principales Resultados a Nivel Localidad del Sistema de Integración Territorial, que refieren al Censo de Población y Vivienda 2010 del Instituto Nacional de Estadística y Geografía (Inegi). Los resultados de Coneval sobre la evolución de la pobreza por ingresos estatal y municipal y la lista de los 125 municipios que conforman la E100x100 tomada de Sedesol (2010).

\subsection{Construcción del Índice de Desarrollo Humano de Localidades}

El IDHL se construye bajo tres lineamientos: considerar las interrelaciones entre las componentes del $\mathrm{DH}$, la dinámica poblacional del país y emplear la mayor cantidad de información disponible. La conceptualización del DH que hemos formulado contempla tres dimensiones: bienestar, educación y salud (cuadro 1).

Un aspecto importante a considerar es que el IDHL fue concebido para ser evaluado a partir de la escala de localidades, con el propósito de tener por primera vez una medida del $\mathrm{DH}$ a este nivel de desagregación. Como consecuencia, la cantidad de información disponible no es tan amplia como a escala de municipios. Por ello es que hemos incluido la componente de bienestar, con la que pretendemos aproximar la componente del ingreso familiar, incluido en el IDH de PNUD. En la figura 1 mostramos la asociación que hay entre ingreso (del IDH de PNUD) y el bienestar que incluimos en el IDHL. Además, la información del ingreso familiar más confiable, usualmente se tiene de una muestra y no de un censo, aunque la información sobre el ingreso en los censos suele tener características que no la hacen confiable (Székely et al., 2007). 


\section{Cuadro 1}

\section{Conceptualización del desarrollo humano}

\begin{tabular}{|c|c|c|}
\hline Dimensión & Componente & Variables empleadas \\
\hline \multirow[t]{3}{*}{ Bienestar } & $\begin{array}{l}\text { Índice de Bienes } \\
\text { (InBie) }\end{array}$ & $\begin{array}{l}\text { Proporción de viviendas particulares habitadas } \\
\text { que disponen de televisión, refrigerador, lava- } \\
\text { dora y computadora. }\end{array}$ \\
\hline & $\begin{array}{l}\text { Índice de Vivienda } \\
\text { (InViv) }\end{array}$ & $\begin{array}{l}\text { Proporción de viviendas particulares habitadas } \\
\text { con piso de material diferente de tierra, con } \\
\text { dos dormitorios o más y que disponen de } \\
\text { excusado o sanitario. }\end{array}$ \\
\hline & $\begin{array}{l}\text { Índice de Servicios } \\
\text { (InSer) }\end{array}$ & $\begin{array}{l}\text { Proporción de viviendas particulares habitadas } \\
\text { que disponen de agua potable, drenaje y } \\
\text { energía eléctrica. }\end{array}$ \\
\hline \multirow[t]{3}{*}{ Educación } & $\begin{array}{l}\text { Índice de Alfabetismo } \\
\text { (InAlf) }\end{array}$ & $\begin{array}{l}\text { Proporción de población alfabetizada de } 8 \text { a } \\
14 \text { años y de } 15 \text { y más años. }\end{array}$ \\
\hline & $\begin{array}{l}\text { Índice de Asistencia } \\
\text { (InAsi) }\end{array}$ & $\begin{array}{l}\text { Proporción de población que asiste a la escue- } \\
\text { la de } 6 \text { a } 11 \text { años, de } 12 \text { a } 14 \text { años y de } 15 \text { a } \\
24 \text { años. }\end{array}$ \\
\hline & $\begin{array}{l}\text { Índice de Escolaridad } \\
\text { (InEsc) }\end{array}$ & $\begin{array}{l}\text { Proporción de población de } 15 \text { y más años } \\
\text { con alguna escolaridad, con educación básica } \\
\text { completa y con algún grado aprobado en nivel } \\
\text { posbásico, y grado promedio de escolaridad. }\end{array}$ \\
\hline \multirow[t]{2}{*}{ Salud } & $\begin{array}{l}\text { Índice de Esperanza de } \\
\text { Vida (InEsp) }\end{array}$ & Esperanza de vida al nacer. \\
\hline & $\begin{array}{l}\text { Índice de Derechoha- } \\
\text { biencia (InDer) }\end{array}$ & $\begin{array}{l}\text { Proporción de población derechohabiente a } \\
\text { servicios de salud. }\end{array}$ \\
\hline
\end{tabular}

Fuente: elaboración propia (2014).

\section{Figura 1}

\section{Comparación del índice de Ingreso (IDH), contra el Índice de Bienestar (IDHL)}
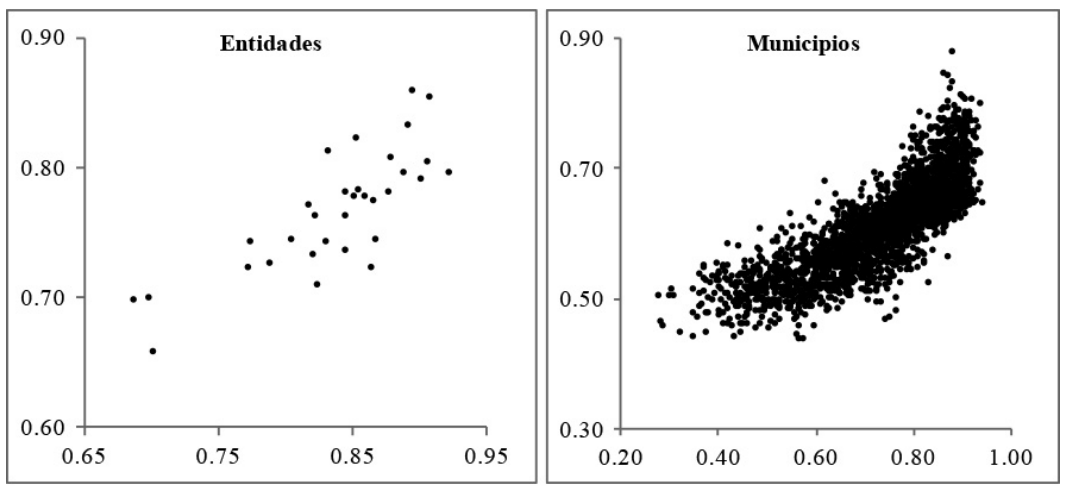

Fuente: elaboración propia con datos de Inegi (2011) y PNUD $(2014,2015)$. 
La forma en que el IDHL se calcula para los municipios se ilustrará con el cálculo del índice de bienes, de la componente de bienestar. Sean los vectores $\mathrm{x}_{1}, \mathrm{x}_{2}, \ldots, \mathrm{x}_{n}$ (uno por cada localidad del país), que consisten de las observaciones de las variables proporción de viviendas particulares habitadas (VPH) que disponen del bien televisión, refrigerador, lavadora y computadora, y la matriz de covarianzas de las variables, calculada a partir de los $\mathrm{x}_{i} i=1,2, \ldots, n$.

Una vez calculada $S$, sean $\mathrm{y}_{1}, \ldots, \mathrm{y}_{m}$ (uno por cada municipio) los vectores con las mismas variables que los $\mathrm{x}_{\mathrm{i}}$, pero que contienen la información de los municipios. La distancia generalizada del vector de observaciones del municipio $j$ al menor punto de desarrollo posible se computa a partir de $\mathrm{S}$. Una distancia generalizada se basa en una matriz $M$ positiva definida, de forma que la distancia entre los vectores $\mathrm{x}$ y $\mathrm{y}$ está dada por $[(x-y) M(x-y)]^{1 / 2}$. Se pueden consultar más detalles en Johnson y Wichern (1999: 30-37).

Por la forma en que están construidas las variables, una localidad (o municipio) alcanza el menor punto de desarrollo posible cuando cada dimensión del vector vale cero. De esta forma, la distancia del municipio está dada por:

$$
d_{j}=\left[\left(\mathbf{y}_{j}-\mathbf{0}\right)^{T} \mathbf{S}\left(\mathbf{y}_{j}-\mathbf{0}\right)\right]^{1 / 2}=\left(\mathbf{y}_{j}^{T} \mathbf{S} \mathbf{y}_{j}\right)^{1 / 2}
$$

donde 0 , es un vector de ceros.

Una vez obtenidas estas distancias, el índice de bienes se construye a partir de la estandarización Mín-Máx de las mismas (Nardo et al., 2005). De esta forma, el índice de bienestar del municipio $j$ se calcula como:

$$
\text { InBie }_{j}=\frac{d_{j}-d_{\text {minn }}}{d_{\text {máx }}-d_{\text {mín }}}=\frac{d_{j}}{d_{\text {máx }}}
$$

Para mantener fijos los umbrales, empleamos como valor mínimo la distancia de un municipio ficticio que tiene el menor grado de desarrollo posible, es decir, $d_{\min }=0$, como este valor no depende de $S$, en cualquier caso, vale cero. Para $d_{\text {max }}$ se toma la distancia a un municipio ficticio con el mayor grado de desarrollo posible, esto es:

$$
d_{\text {máx }}=\left(\mathbf{1}^{T} \mathbf{S} 1\right)^{1 / 2}
$$

donde 1 es un vector de unos (la proporción de VPH que poseen el correspondiente bien es 1 para todos los bienes considerados).

Como mencionamos anteriormente, el IDH es, operativamente, el promedio de tres indicadores y debido a esto no son consideradas las 
relaciones entre las dimensiones del $\mathrm{DH}$, en el sentido de que un incremento en alguna de sus componentes tiene un efecto en el índice final independiente de los demás indicadores. Sin embargo, el DH es un proceso complejo que se simplifica demasiado al no considerar relaciones entre sus partes.

Por ello, la forma operativa del IDHL que planteamos es a partir de una distancia generalizada, definida a través de la matriz de covarianzas de los valores de los indicadores de las dimensiones del DH, la cual se estandariza para obtener así el índice. Esto tiene como consecuencia que las relaciones entre cada par de dimensiones del $\mathrm{DH}$ quedan cuantificadas por la covarianza entre sus indicadores.

El segundo criterio usado en la construcción del IDHL toma en cuenta la dinámica poblacional del país, con esto nos referimos a que la medida está diseñada para identificar las diferencias en una escala menor. El IDHL de los municipios es calculado a partir de la información de las localidades, esto es, las matrices de covarianzas empleadas en el cálculo del índice de municipios fueron calculadas con la información de las localidades, ya que a mayor nivel de desagregación (como lo es a nivel localidad) se tiene una mayor variabilidad en las características del DH y esto implica un mejor detalle de las desigualdades en $\mathrm{DH}$ presentes en el país.

Por último, cuando decimos que en el IDHL se emplea la mayor cantidad de información disponible, no nos referimos a que usamos todas las variables disponibles, sino que consideramos las variables que de algún modo, sirven como indicadores de las dimensiones del DH y se encuentran para cada nivel de desagregación (localidades, municipios, entidades, nacional). En total son 21 las variables usadas en el cálculo del IDHL.

Por esta construcción, el indicador propuesto resulta ser más general y a la vez más preciso para la medición del $\mathrm{DH}$. También, contempla las 11 variables utilizadas en el IRS, sólo que en un sentido positivo y no como carencias. Por esta última característica, el IDHL incluye una medida del no-rezago social.

A excepción del grado promedio de escolaridad y la esperanza de vida, las variables empleadas son proporciones y por lo tanto actúan como indicadores. Para las dos excepciones se utilizó una estandarización MínMáx para transformarlas en índices. Esta estandarización consiste en hacer una transformación de la forma

$$
I_{i}=\frac{x_{i}-x_{\min }}{x_{\max }-x_{\min }}
$$

donde $x_{i}$ es el valor observado de la variable en el territorio $i, \mathrm{y} x_{\max } \mathrm{y}$ $x_{\text {min }}$ son los valores máximo y mínimo observados de la variable de interés. 
Los umbrales empleados para el grado promedio de escolaridad fueron los observados, 0 y 18.25 años; en el caso de la esperanza de vida los valores fueron 25 y 85 años.

Con este procedimiento se calcula cada uno de los índices, excepto el de salud, en el que para su construcción se empleó el proceso jerárquico analítico considerando nueve veces más importante la esperanza de vida que la derechohabiencia. Esto debido a que la información empleada para evaluar salud no está disponible a nivel localidad y se hace un ajuste estimando la esperanza de vida de una localidad con la esperanza de vida del municipio al que pertenece.

\section{Resultados}

Como se planteó en la introducción, el propósito de este trabajo es proponer un índice para medir el $\mathrm{DH}$ en los municipios del país y utilizarlo para verificar si los municipios que están considerados en la E100x100 son los 125 con menor DH. Las diferencias observadas entre los grupos de municipios nos permitieron obtener conclusiones acerca de los objetivos que hemos planteado.

Considerando el IDHL como medida del DH, seleccionamos 125 municipios según la información de 2010 con los siguientes criterios:

i) 100 municipios con menor IDHL;

ii) Del resto, 25 municipios con menor IDHL de los que tienen más de $60 \%$ de población en pobreza alimentaria.

A partir de esta selección y la de 2007, se identificaron los municipios según las coincidencias o discrepancias que presentaron. Para ello, formamos tres grupos de municipios:

i) Municipios que deben permanecer en la estrategia (Grupo A): municipios que aparecen en ambas selecciones.

ii) Municipios que deberían pertenecer a la estrategia (Grupo B): municipios que no son seleccionados en 2007 pero que sí lo son en 2013.

iii) Municipios que deberían dejar de pertenecer a la estrategia (Grupo C): municipios que fueron seleccionados en 2007 pero que ya no lo fueron en 2013.

De lo anterior se sigue que la selección de 2007 estuvo compuesta por los grupos A y C, y la selección en 2013 está formada por los grupos A y B. 
Las diferencias entre ambos conjuntos se podrían atribuir a dos hechos. El primero, sostenido por nosotros como más plausible, es que a través de este periodo la situación de los municipios cambió, en algunos casos de manera favorable. La segunda causa es el cambio en el instrumento de medida (IDH por IDHL) para la selección de los municipios y con ello, el cambio en los criterios de selección. Se debe tener en cuenta que el IDHL es un indicador más general, incluso contiene una medida de norezago social y está expresamente construido para las condiciones sociales de México. Por este motivo, consideramos que los cambios, al pasar del IDH al IDHL, son significativos.

Según los resultados obtenidos, en todos los grupos analizados existen serias carencias en cuanto a la disponibilidad de bienes, acceso a servicios básicos, infraestructura de la vivienda, escolaridad y derechohabiencia a servicios de salud. Sin embargo, en algunos casos los problemas resultan ser más serios que en otros, lo que nos permite identificar cuáles municipios deben ser prioritarios y cuáles deben pasar a segundo plano.

En el cuadro 2 se muestran los municipios por entidad federativa que fueron seleccionados para la E100x 100 en 2007 y los del 2013. Como se esperaba, se encontraron diferencias tanto en las entidades como en los municipios. Según los criterios de selección en 2013, los municipios de diez entidades federativas fueron considerados más vulnerables, mientras que en la E100x100 (2007) sólo se consideran siete. Las nuevas entidades consideradas son: Chihuahua, Jalisco y San Luis Potosí.

También existen cambios en el número de municipios por entidad y por tanto, el número de personas consideradas en la E100x100. La población en la nueva selección es de 1,631,216 habitantes, es decir, 4,2\% mayor a la considerada en la estrategia.

A continuación describimos detalladamente la conformación de cada uno de los grupos. Debido a que los índices empleados constituyen una medida ordinal, cuando se hace referencia a ellos se usa la mediana como medida de tendencia central. Para el caso de los porcentajes se usan promedios (las listas de los municipios que pertenecen a cada grupo se muestran en el Anexo).

\subsection{Municipios que deben permanecer en la E100x100 (Grupo A)}

Este grupo está conformado por 84 municipios de siete entidades: Chiapas, Durango, Guerrero, Nayarit, Oaxaca, Puebla y Veracruz; su población es de 1,188,767 habitantes, que corresponden al $76 \%$ del total de la E100x100. El porcentaje promedio de población en pobreza alimentaria es de 71,2 por ciento. 


\section{Cuadro 2 \\ Municipios identificados como más vulnerables por entidad federativa}

\begin{tabular}{|c|c|c|c|c|c|}
\hline \multicolumn{3}{|c|}{ Selección 2007 (E100x100) } & \multicolumn{3}{|c|}{ Selección 2013} \\
\hline Entidad & Mpios. & Población & Entidad & Mpios. & Población \\
\hline Chiapas & 20 & 483,887 & Chiapas & 17 & 491,086 \\
\hline Durango & 1 & 33,396 & Chihuahua & 5 & 134,093 \\
\hline Guerrero & 21 & 411,979 & Durango & 1 & 33,396 \\
\hline Nayarit & 1 & 34,300 & Guerrero & 19 & 355,075 \\
\hline Oaxaca & 58 & 294,425 & Jalisco & 1 & 18,084 \\
\hline Puebla & 9 & 104,629 & Nayarit & 1 & 34,300 \\
\hline Veracruz & 15 & 202,401 & Oaxaca & 67 & 342,925 \\
\hline \multirow[t]{4}{*}{ Total } & 125 & $1,565,017$ & Puebla & 3 & 47,309 \\
\hline & & & San Luis Potosí & 2 & 59,258 \\
\hline & & & Veracruz & 9 & 115,690 \\
\hline & & & Total & 125 & $1,631,216$ \\
\hline
\end{tabular}

Fuente: elaboración propia con información de Inegi (2011) y Coneval (2013b).

En este grupo, 45 municipios tienen un DH bajo, el resto se ubica en un nivel medio-bajo. ${ }^{1}$ Las principales carencias de este grupo son: disponibilidad de bienes, escolaridad y derechohabiencia a servicios de salud (cuadro 3).

\section{Cuadro 3}

\section{Resumen de índices del Grupo A}

\begin{tabular}{cccc}
\hline Bienestar & Bienes & Servicios & Vivienda \\
\hline 0,4443 & 0,1713 & 0,5319 & 0,6856 \\
Educación & Alfabetismo & Asistencia & Escolaridad \\
\hline 0,5764 & 0,7294 & 0,6608 & 0,3026 \\
Salud & Derechohabiencia & Esperanza de vida & Desarrollo humano \\
\hline 0,7164 & 0,5576 & 69,0 & 0,5051 \\
\hline
\end{tabular}

Fuente: elaboración propia con información de Inegi (2011).

En promedio, 62,4\% de las VPH no disponen de televisión (TV), $84,4 \%$ de refrigerador (REF), $96,1 \%$ de lavadora (LAV) y $98,8 \%$ de computadora (PC); 32.8\% tienen piso de tierra (PIS), 63,1\% tienen solamente un dormitorio (DOR) y $19 \%$ no disponen de excusado o sa-

${ }^{1}$ Esta es la clasificación empleada por el Conapo (2001), en ella se consideran los siguientes niveles de DH: bajo si el valor del índice es menor que 0,50; medio-bajo si el índice está entre 0,5 y 0,65; medio-alto si el índice está entre 0,65 y 0,80; y alto si el índice es mayor que 0,80. En todos los casos los intervalos incluyen los extremos inferiores. 
nitario (EXC); $51,7 \%$ están sin agua potable (AG), 74,4\% sin drenaje (DR) y $17 \%$ sin electricidad (EL), gráfica 1.

La situación educativa es la siguiente: en promedio $18,1 \%$ de niños (8 a 14 años, ALF1) y 36,3\% de adultos (15 años y más, ALF2) son analfabetas; 6,8\% de la población de 6 a 11 años (ASI1), 15,5\% de la población de 12 a 14 años (ASI2) y 73,4\% de la población de 15 a 24 ańos (ASI3) no asiste a la escuela; 30,9\% de adultos no tiene escolaridad (ESC1), 81,2\% tiene educación básica incompleta (ESC2), 92,4\% no tiene algún grado aprobado en educación postbásica (ESC3), y el grado promedio de escolaridad es de 4,27 años (gráfica 1).

\section{Gráfica 1}

Porcentaje de viviendas con carencias de los municipios del Grupo A

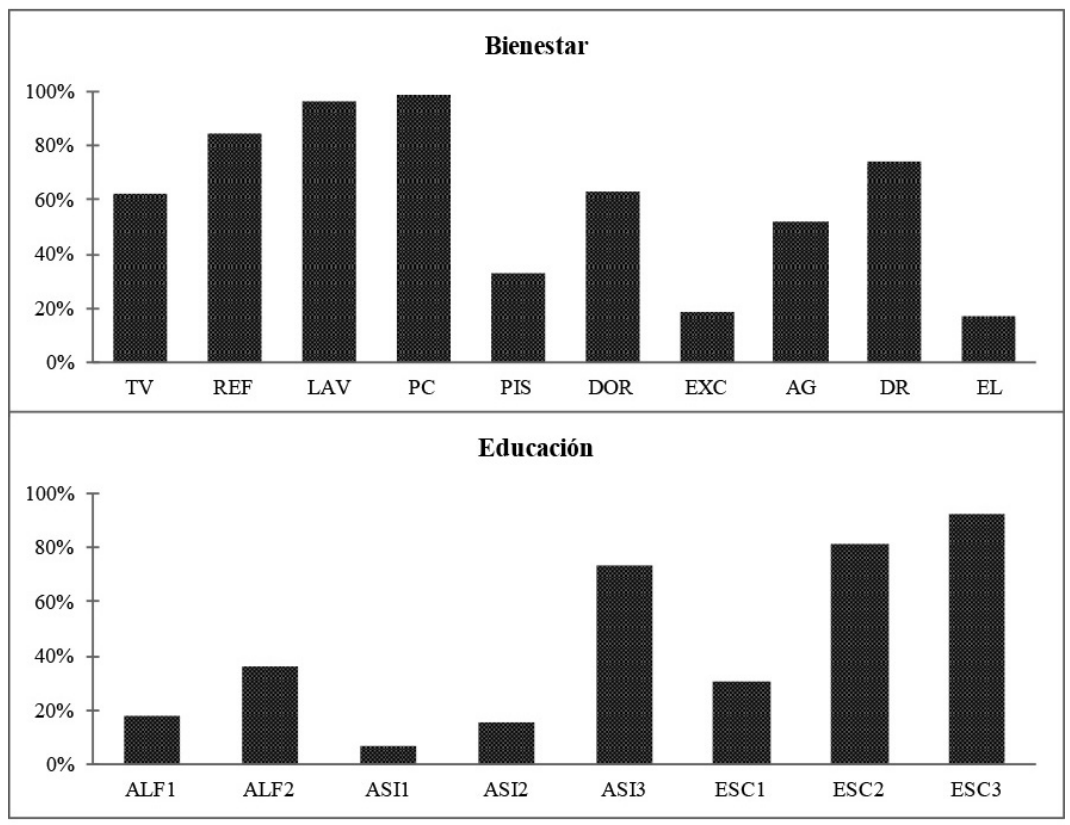

Fuente: elaboración propia con información de Inegi (2011).

Por último, 44,2\% de la población no tiene derechohabiencia a servicios de salud y la esperanza de vida promedio es de 69 años.

\subsection{Municipios que deberían salir de la E100X100 (Grupo B)}

Este grupo está conformado por 41 municipios de cinco entidades: Chiapas, Guerrero, Oaxaca, Puebla y Veracruz; con una población de 376,250 habitantes, que representa $24 \%$ de E100x100. El porcentaje promedio de población en situación de pobreza alimentaria es de 63,9 por ciento. 
Las principales carencias de este grupo son: disponibilidad de bienes y escolaridad. A pesar de su situación, todos los municipios de este grupo tienen un DH medio-bajo a excepción de dos: San Francisco Tlapalcingo en Oaxaca y Francisco León en Chiapas, los cuales tienen un DH medioalto. Según estos resultados es claro que, de acuerdo con el criterio del $\mathrm{DH}$, la condición de estos municipios es mejor que la de los municipios del Grupo A (cuadro 4).

\section{Cuadro 4 \\ Resumen de índices del Grupo B}

\begin{tabular}{cccc}
\hline Bienestar & Bienes & Servicios & Vivienda \\
\hline 0,5420 & 0,2477 & 0,6946 & 0,7557 \\
Educación & Alfabetismo & Asistencia & Escolaridad \\
\hline 0,5865 & 0,7593 & 0,6765 & 0,3045 \\
Salud & Derechohabiencia & Esperanza de vida & Desarrollo humano \\
\hline 0,7259 & 0,6414 & 69,6 & 0,5662 \\
\hline
\end{tabular}

Fuente: elaboración propia con información de Inegi (2011).

Como se mencionó, los municipios de este grupo tienen las mayores carencias en disponibilidad de bienes, puesto que el porcentaje de viviendas que carecen de un bien supera $45 \%$ en cualquiera de los casos. Además de esta característica, se tienen problemas graves de hacinamiento y acceso a drenaje, pues $60,8 \%$ de las VPH tienen únicamente un dormitorio y $50,4 \%$ no tiene acceso a drenaje. No obstante, en todas las condiciones mencionadas están mejor que el Grupo A. En las demás características del bienestar, los porcentajes de viviendas con carencias son menores a $30 \%$ (gráfica 2).

La situación educativa muestra un mejor panorama pero sólo en el caso de los niños, ya que casi 90\% saben leer y escribir, mientras que sólo dos terceras partes de los adultos están alfabetizados. Esta diferencia también se manifiesta en la asistencia escolar, ya que en los menores es casi tres veces más alta que en el caso de los adultos. Otra parte del problema en la educación de estos últimos radica en que hay un porcentaje alto sin instrucción alguna, pero más alto aún es el porcentaje sin educación básica completa y sin grados aprobados en el nivel posbásico (gráfica 2).

Por último, en promedio $35,9 \%$ de población no tiene derechohabiencia a servicios de salud, no obstante existen tres municipios en los que el porcentaje de población derechohabiente es mayor a 90\%, estos son San Francisco Tlapalcingo y Santiago Texcalcingo en Oaxaca y Xoxocotla en Veracruz. La esperanza de vida promedio es de 69,6 años, aunque hay una diferencia de 13,4 años entre los municipios con la máxima y la mínima esperanza de vida de este grupo. 


\section{Gráfica 2}

\section{Porcentaje de viviendas con carencias de los municipios del Grupo B}

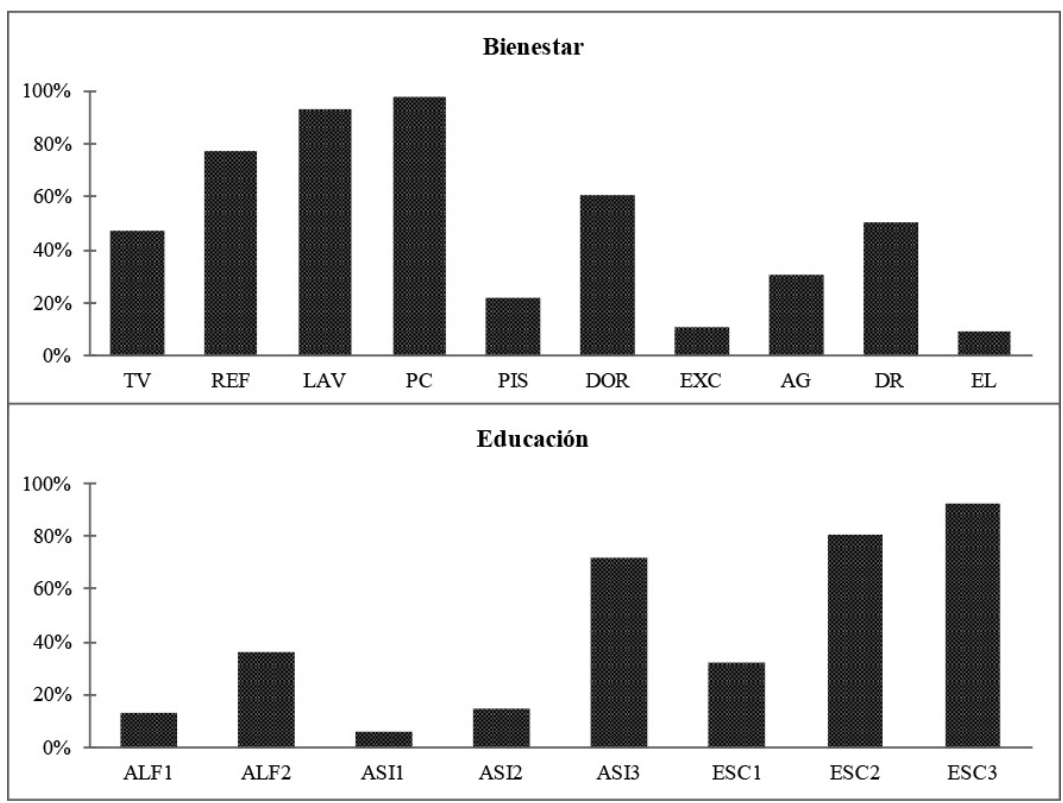

Fuente: elaboración propia con información de Inegi (2011).

\subsection{Municipios que deben considerarse en la E100X100 (Grupo C)}

Este grupo está formado por 41 municipios de seis entidades: Chiapas, Chihuahua, Guerrero, Jalisco, Oaxaca y San Luis Potosí. Su población es de 442,449 habitantes, que corresponde a $27 \%$ de la seleccionada en 2013. El porcentaje promedio de población en pobreza alimentaria es de 62,9\%. En este grupo 12 municipios tienen un $\mathrm{DH}$ bajo, el resto tiene un nivel medio-bajo (cuadro 5).

\section{Cuadro 5}

Resumen de índices del Grupo C

\begin{tabular}{cccc}
\hline Bienestar & Bienes & Servicios & Vivienda \\
0,426 & 0,2113 & 0,4500 & 0,6908 \\
Educación & Alfabetismo & Asistencia & Escolaridad \\
0,6403 & 0,8332 & 0,7023 & 0,3652 \\
Salud & Derechohabiencia & Esperanza de vida & Desarrollo humano \\
0,744 & 0,5333 & 70,6 & 0,5107 \\
\hline
\end{tabular}

Fuente: elaboración propia con información de Inegi (2011). 
El nivel de bienestar de los municipios de este grupo es similar al de los del Grupo A. En ambos se tiene una baja disponibilidad de bienes, cuyas carencias más notables son la falta de televisión y refrigerador; los porcentajes de viviendas en las que no figuran estos bienes son $58,5 \%$ y $79,8 \%$, respectivamente. En cuanto a infraestructura de las viviendas los resultados no son significativamente diferentes a los de los otros casos. Sin embargo, la peor situación se presenta en el acceso a servicios básicos, puesto que $58,5 \%$ de las viviendas no cuenta con agua potable, $80,8 \%$ carece de drenaje y $23,1 \%$ de electricidad, porcentajes superiores a los observados en los grupos A y B (gráfica 3).

La situación de la educación de los municipios de este grupo es similar al del grupo anterior, de hecho, la condición educativa muestra un comportamiento homogéneo en los tres grupos. En el caso de la salud, puesto que el valor de la esperanza de vida de este grupo es de 70,6 años, presenta cierta similitud con los otros dos, en consecuencia el Índice de Salud presenta aproximadamente los mismos valores. A pesar de este hecho, la derechohabiencia de este grupo es la más infame de los tres grupos, ya que 46,7\% de la población no es derechohabiente (gráfica 3).

\subsection{Comparación de los grupos}

Resulta más fácil identificar gráficamente las diferencias entre los distintos grupos, por lo que en la gráfica 4 se presentan los perfiles de los tres grupos, donde se aprecian los aspectos más relevantes, mostrados con anterioridad en los cuadros 3, 4, y 5 .

Podemos identificar que los grupos A y $\mathrm{C}$ tienen un IDHL similar, 0,5051 y 0,5107, respectivamente; pero ambos menores al del Grupo B, 0,5662. Por sí mismo, este hecho, es suficiente para sostener que los municipios del Grupo C deben pasar a ser prioritarios en lugar de los del Grupo B.

Presentamos una comparación de los grupos $\mathrm{B}$ y $\mathrm{C}$, donde se excluye al Grupo A, debido al carácter de su constitución, puesto que está formado por municipios que ya son prioritarios. Sólo es importante mencionar que su condición es en todos los aspectos, más desfavorable que la del Grupo B.

El Grupo B tiene una mejor situación en los aspectos de bienestar: disponibilidad de bienes, infraestructura de la vivienda y acceso a servicios, siendo esta última la diferencia más notable. Respecto a las carencias, en cuanto al acceso al agua potable, en promedio $30,4 \%$ de las viviendas del Grupo B carecen de este servicio, contra 58,5\% en el caso del Grupo C, en drenaje es de $50,4 \%$ contra $80,8 \%$, y en electricidad, es $9,4 \%$ contra 23,1 por ciento. 


\section{Gráfica 3}

Porcentaje de viviendas con carencias de los municipios del Grupo C

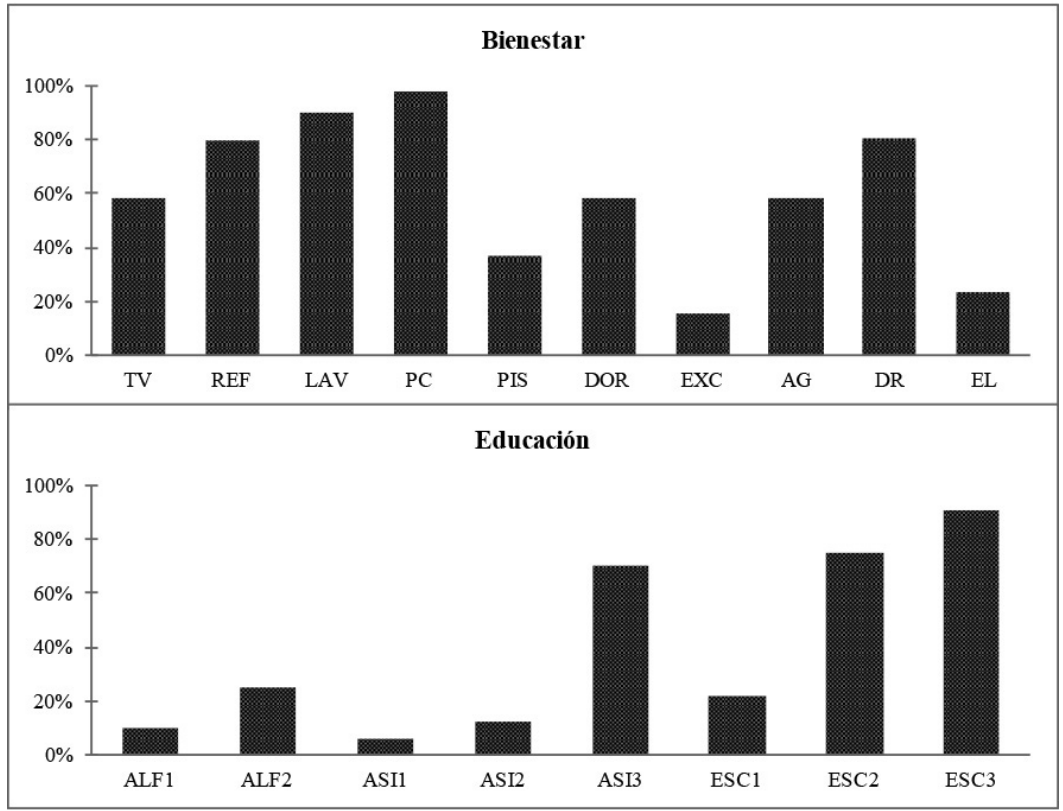

Fuente: elaboración propia con datos de Inegi (2011).

\section{Gráfica 4}

\section{Perfil de los grupos de municipios vulnerables}

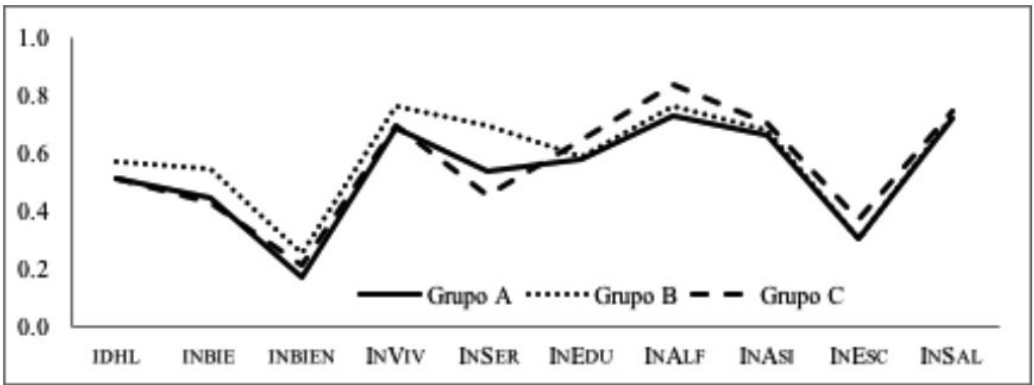

Fuente: elaboración propia con información de Inegi (2011).

Por otro lado, el Grupo C tiene una mejor situación en educación y en salud. En el caso del alfabetismo, la diferencia es mayor en adultos que en la población infantil. En asistencia, los porcentajes no son tan disimiles, con diferencias menores a 3\% en todos los casos. En escolaridad, la discrepancia más evidente está en el porcentaje de población adulta sin instrucción alguna, el grado promedio de escolaridad es 0,9 años mayor 
en el tercer grupo. A pesar de lo antes descrito el IDHL del Grupo C es menor al del Grupo B y de ahí que se reconozca la condición más desfavorable para el primero.

Podemos considerar que los resultados anteriores son, en términos generales, congruentes con los presentados al realizar la selección para la E100x 100. Ya que independientemente del grupo al que pertenezcan, los municipios seleccionados tienen perfiles de $\mathrm{DH}$ bastante similares. Las diferencias encontradas también se muestran congruentes a lo esperado en cuanto al cambió de la condición de los municipios de la E100x100 en el transcurso de este periodo.

\section{Discusión}

El proceso de construcción de un índice social se puede dividir en tres etapas. De acuerdo con Herrero et al. (2010), primero se debe delimitar adecuadamente la característica social que se desea cuantificar, después seleccionar las variables adecuadas para medirla $y$, finalmente, exponer la forma en que el índice será calculado. El enfoque que describen Foa y Tanner (2012) coincide con estos autores, aunque dan por sentado que la característica que se desea medir ya se ha especificado en el aspecto que se pretende calcular.

Tomando en cuenta la primera descripción del proceso, vemos que la etapa de selección del objeto de estudio corresponde a los teóricos y especialistas en el área, puesto que serán ellos lo que definan los aspectos específicos de la característica que se desea estudiar. En el presente trabajo, hemos tomado la concepción de DH de Sen, que consiste en describir el DH como las opciones con las que las personas cuentan para el desarrollo de sus capacidades (PNUD, 1990).

La selección de variables que aporten a la medición de la característica seleccionada, corresponde a los especialistas en el área y a los expertos en medición social. Por un lado, los especialistas han de seleccionar cuáles serán las variables a considerar en la medida en que aportan algo a la medición del objetivo. Después, los expertos en medición social se encargarán de determinar la calidad de la información disponible. Foa y Tanner (2012) señalan también que mientras más impreciso sea el concepto y pocas las fuentes de información, es más probable que se requiera una cantidad considerable de variables para construir un indicador adecuado. Sobre este punto, hemos considerado los principios que establece Sen (PNUD, 1990) para la medición del DH, que son los mismos que $\mathrm{Na}-$ ciones Unidas usa en la construcción del IDH. Sen (1985) señala que el $\mathrm{DH}$ debe ser medido a través del acceso a recursos para una vida digna, a una educación de calidad y a opciones para gozar de una vida larga y 
saludable. Con esta base hemos seleccionado las variables a incluir en el índice, considerando la mayor cantidad de información confiable a escala de localidades.

La última etapa es quizá donde se concentra el mayor número de aportaciones; típicamente los índices son considerados como promedios ponderados, por lo que la selección del método de cálculo se traduce en la elección de los pesos (Mishra, 2007). Por ejemplo, en el "Manual para la construcción de indicadores compuestos" de Nardo et al. (2005) se enlistan al menos ocho criterios diferentes para calcular indicadores.

Nuestra propuesta se fundamenta en el uso de una métrica estadística, también llamada distancia de Mahalanobis. Este concepto fue introducido por Mahalanobis (1936) como una generalización de la distancia euclidiana a partir del estudio de la distribución normal multivariada (Johnson y Wichern, 1999). Se pueden consultar los trabajos de McLachan (2000) y de De Maesschalck et al. (2000) para una explicación e interpretación de esta métrica.

El IDH ha sido cuestionado desde su publicación en 1990. Desde ser un indicador redundante (McGillivray, 1991), hasta cuestionar la validez de combinar dimensiones incomparables en un sólo indicador (Michinaka, 2008). De la misma forma, también han sido presentadas propuestas de mejoras al cálculo del índice. Se expone la idea de un indicador más general basado en una distancia (Noorbakhsh, 1998), uno que tome en cuenta rendimientos decrecientes (Chakravarty, 2003) e incluso PNUD ha cambiado la forma de agregación a una media geométrica (PNUD, 2010) cuyos detalles los presenta Zambrano (2011). Una revisión de modificaciones que se han hecho al IDH se puede ver en Klugman et al. (2011). Sin embargo, en ningún trabajo se ha planteado medir el DH a partir de una distancia generalizada como lo hacemos en éste.

El índice propuesto obtuvo una autovaloración positiva al compararlo con algunos de los criterios que establecen Hagerty et al. (2001) para evaluar índices de calidad de vida. El IDHL es un índice de desarrollo humano y forma parte de la calidad de vida; entre sus características: tener un propósito claro, es decir, medir las opciones con las que las personas cuentan para el desarrollo de sus capacidades, estar basado en una teoría establecida, tal como la teoría de capacidades de Sen (1985), y construirse a partir de series de tiempo que permitan un monitoreo y control (censos y conteos de población y vivienda), así como estar disponible para la planeación en todos los niveles de agregación. Cabe decir que el IDHL está disponible para todas las escalas territoriales; en este sentido, pudiera posicionarse como una herramienta útil, al basarse en argumentos estadísticos matemáticos sólidos y con una conceptualización válida que se sostiene en la teoría del desarrollo humano. Además, se 
construyó con el propósito de servir como indicador para el monitoreo espacial y temporal de la población en cuanto a su desarrollo humano.

Dado el carácter interno del indicador que aquí mostramos, no es posible comparar directamente los resultados obtenidos, por lo que nos concentraremos en los trabajos nacionales. Para valorar la consistencia de los resultados que proporciona el IDHL, los hemos comparado con resultados de otros indicadores disponibles. Consideraremos el IRS del Coneval (2011b), el Índice de Marginación (IM) del Conapo (2011) y el IDH de PNUD (2014, 20I5), evaluados a nivel municipal y estatal en 2010.

En la figura 2 (izquierda) mostramos la comparación a escala estatal. Los resultados indican una marcada asociación entre los distintos índices y el IDHL, puesto que los coeficientes de correlación son -0,98, -0,96 y 0,83 , respectivamente. La asociación negativa del IDHL con el IRS y el IM se da porque el DH está asociado con factores positivos, mientras que el rezago social y la marginación están vinculados con factores negativos. Adicionalmente, podemos observar en la gráfica como los cuatro indicadores coinciden en señalar la brecha que existe entre Chiapas, Guerrero y Oaxaca y el resto de los estados del país, señalados por los tres conjuntos de puntos en la parte superior izquierda.

En la figura 2 (derecha) se presenta una comparación similar, pero para la escala municipal. En este caso, la asociación entre los índices y el IDHL también es alta, los coeficientes de correlación son -0,98, -0,94 y 0,84 , respectivamente. Estos resultados confirman la validez y consistencia de las mediciones del DH que proporciona el IDHL.

Los resultados anteriores señalan que el IDHL proporciona resultados consistentes a los que proporcionan otros índices. Asimismo, nos permiten exhibir como la correspondencia entre el IDHL y el IDH es menor que con el IRS y el IM, esto proporciona evidencia empírica para sostener que la métrica aquí propuesta refleja de una mejor manera las condiciones de la población mexicana y una evidencia analítica, como se muestra en el cuadro 6, si se observan las correlaciones respectivas del IDH y el IDHL con los otros índices.

Por otro lado, una actualización de los índices de marginación y pobreza con la metodología propuesta es una tarea futura que permitirá incorporar en ellos las interacciones entre las variables que los componen que, en la actualidad, no se consideran, lo cual exhibirá la fortaleza de la métrica usada. 


\section{Figura 2}

Comparación IDHL vs. IRS e IM a escala estatal y municipal, 2010

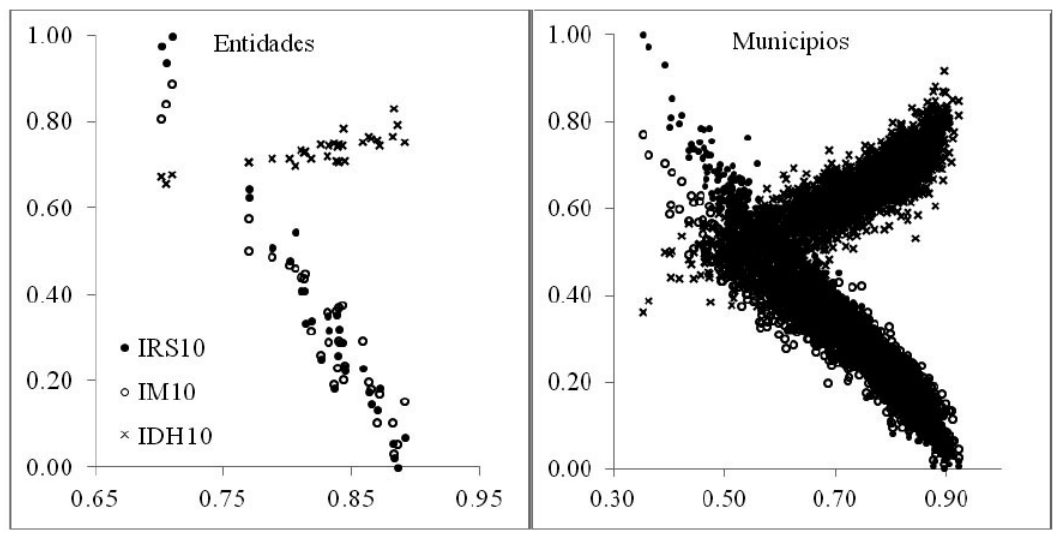

Fuente: Elaboración propia con datos de Conapo (2011), Coneval (2011b) y PNUD (2014, 2015).

\section{Cuadro 6}

Coeficientes de correlación a escala de municipios, 2010

\begin{tabular}{lccc}
\hline & ISR10 & IM10 & IDH10 \\
\hline IDHL10 & $-0,98$ & $-0,94$ & 0,84 \\
ISR10 & & 0,96 & $-0,87$ \\
IM10 & & & $-0,89$ \\
\hline
\end{tabular}

Fuente: Elaboración propia con datos del Conapo (2011), Coneval (2011b) y PNUD (2014).

\section{Conclusiones}

En este artículo nos hemos circunscrito al DH y a la E100x100; sin embargo, los resultados obtenidos pueden ser aplicados a cualquier característica de la sociedad y programa social; principalmente cuando la población objetivo sea definida a partir de un ordenamiento de alguna medida social. Dado que la intensidad de la afectación o desventaja que presentan ciertas poblaciones dependerá siempre del instrumento de medición que se utilice. Por ello, al contar con herramientas de ordenamiento bien sustentadas, podremos obtener mejores resultados que sirvan a los tomadores de decisiones para lograr el mejor uso de los recursos públicos.

Como se comentó, la E100x100 tiene el objetivo de focalizar los programas sociales sobre la población de los 125 municipios más desfavorecidos, esto con el objetivo de mejorar su calidad de vida y bienestar. 
Después de seleccionar un grupo de municipios que en 2010 se encontraban en las condiciones más extremas de vulnerabilidad, y observar cambios en la composición de este grupo y el universo de acción, pudimos explicar las causas de estos cambios, a partir de la medida de desarrollo humano que hemos aplicado.

Con los resultados obtenidos es claro que hay diferencias entre la selección de municipios hecha en 2007 y la que exhibimos en este estudio. Tales discrepancias se deben principalmente al bienestar, que es medido por la disponibilidad de bienes, infraestructura y acceso a servicios de las viviendas. En los casos de educación y salud, si bien hay diferencias, no son un factor significativo para determinar qué municipios están en mejores condiciones que otros.

La conclusión anterior está de acuerdo con el siguiente hecho: los indicadores empleados para medir el desarrollo humano consideran únicamente necesidades básicas del mismo, y que en los niveles en los que se encuentran los habitantes de los territorios mencionados, los aspectos del acceso a recursos para un nivel de vida decente (el ingreso, recursos o patrimonio) se presentan como condición diferenciadora del desarrollo. De esta manera se justifica la determinación de tres grupos de municipios: los que deben permanecer como prioridad, los que deben pasar a un segundo plano y los que deben integrarse como prioritarios a la estrategia.

Actualmente existe una consulta donde se evalúa el desempeño de la E100x100, dicho documento fue publicado como parte de la respuesta a la solicitud de la Comisión Intersecretarial de Desarrollo Social para elaborar una evaluación que permitiera identificar resultados y áreas de oportunidad (Coneval, 2012). En el mencionado estudio se realiza una evaluación del impacto de la estrategia, no se debate acerca de la vigencia del conjunto de municipios que están siendo beneficiados por ella, por lo cual este trabajo también resulta relevante.

Los resultados obtenidos permiten concluir que el objetivo de la E100x100 se está dejando de cumplir. Además, fue posible determinar que las diferencias más notables se encuentran en bienestar (disponibilidad de bienes e infraestructura y acceso a servicios en las viviendas), en segundo lugar en educación (alfabetismo, asistencia escolar y logro educativo), y en último lugar, en la situación de la salud.

En conclusión, la contribución de este trabajo se centró en la construcción de un índice de desarrollo humano focalizado para las localidades, el cual captura las diferencias que existen en las personas que habitan los lugares más pequeños de nuestra partición territorial y las traslada a la escala municipal que se usa en la E100x100. El propósito se centra en medir el desarrollo humano en las condiciones singulares de nuestro país, fraccionado territorialmente pero aglutinado poblacionalmente, de modo 
que represente la especificidad de nuestros espacios y, principalmente, las circunstancias de los habitantes en esos espacios.

Una limitación de la conclusión anterior es que la ventaja en patrimonio que tienen los municipios del Grupo B se debe a la disponibilidad de bienes, aunque el acceso a servicios (agua potable, drenaje y energía eléctrica) y, en segundo término, la infraestructura de las viviendas, desempeñan un papel importante en esta diferenciación. Y es que al especificar la relación entre acceso a servicios y el acceso a recursos para una vida decente, se encuentra que el acceso a servicios es una condición necesaria, pero no suficiente, para el desarrollo.

$\mathrm{Al}$ elaborar un instrumento propio para el país, nos orientamos a examinar la situación de los municipios seleccionados en la E100x100 en el 2007 para señalar la conveniencia de mantenerlos o exhibir la información básica, y de esta manera proponer la exclusión de aquellos que han mejorado significativamente en relación con otros que ahora se muestran en situaciones que los hacen más elegibles para sustituir a aquellos.

$\mathrm{Al}$ emplear una medida diseñada específicamente para evaluar el $\mathrm{DH}$ en México se obtienen mejores resultados, porque se consideran dos elementos esenciales en un Estado, las consecuencias, tanto por la dispersión de sus territorios como por el estructural aglutinamiento de su población, además se emplea para su cálculo más información. Esta última característica, lejos de proporcionar un indicador redundante, responde a la complejidad intrínseca de un proceso como el $\mathrm{DH}$.

La metodología con la que se construyó el IDHL puede ser empleada para considerar índices de características de diversa naturaleza, por ejemplo, el IRS que el Coneval construyó a partir de la técnica estadística de componentes principales argumentando que de esa forma el Conapo construyó el Índice de Marginación (Coneval, 2007). Nuestra propuesta exhibe empíricamente una correspondencia aceptable y robusta con el conocimiento que se tiene del desarrollo humano en los territorios de México y tales características se pueden apreciar en su propiedad discriminatoria, al señalar las dimensiones en las que los municipios son semejantes y aquellas en las que se diferencian. La característica central de la métrica es que considera las diversas interacciones que las variables tienen entre sí, de modo que no sólo toma en cuenta las contribuciones de cada una de ellas. Queda como línea de trabajo, la investigación de las propiedades teóricas de indicadores construidos a partir de esta métrica estadística. 


\section{Anexo \\ Municipios por grupo}

\section{Cuadro 7 \\ Municipios del Grupo A}

\begin{tabular}{|c|c|c|c|}
\hline Entidad & Municipio & Entidad & Municipio \\
\hline Chiapas & Amatenango del Valle & Oaxaca & Coicoyán de las Flores \\
\hline Chiapas & Chalchihuitán & Oaxaca & Eloxochitlán de Flores Magón \\
\hline Chiapas & Chamula & Oaxaca & Huautepec \\
\hline Chiapas & Chanal & Oaxaca & Magdalena Peñasco \\
\hline Chiapas & Chenalhó & Oaxaca & Mazatlán Villa de Flores \\
\hline Chiapas & Chilón & Oaxaca & San Agustín Loxicha \\
\hline Chiapas & Huixtán & Oaxaca & San Andrés Paxtlán \\
\hline Chiapas & Larráinzar & Oaxaca & San Antonio Sinicahua \\
\hline Chiapas & Mitontic & Oaxaca & San José Lachiguiri \\
\hline Chiapas & Pantelhó & Oaxaca & San José Tenango \\
\hline Chiapas & Sitalá & Oaxaca & San Juan Coatzóspam \\
\hline Chiapas & Zinacantán & Oaxaca & San Juan Comaltepec \\
\hline Chiapas & San Juan Cancuc & Oaxaca & San Juan Petlapa \\
\hline Chiapas & Aldama & Oaxaca & San Lorenzo Texmelúcan \\
\hline Chiapas & Santiago el Pinar & Oaxaca & San Lucas Camotlán \\
\hline Durango & Mezquital & Oaxaca & San Lucas Zoquiápam \\
\hline Guerrero & Alcozauca de Guerrero & Oaxaca & San Marcial Ozolotepec \\
\hline Guerrero & Atlamajalcingo del Monte & Oaxaca & San Martín Itunyoso \\
\hline Guerrero & Atlixtac & Oaxaca & San Miguel Coatlán \\
\hline Guerrero & Copalillo & Oaxaca & San Miguel Mixtepec \\
\hline Guerrero & Copanatoyac & Oaxaca & San Miguel Peras \\
\hline Guerrero & Malinaltepec & Oaxaca & San Pablo Cuatro Venados \\
\hline Guerrero & Metlatónoc & Oaxaca & Santa Ana Ateixtlahuaca \\
\hline Guerrero & Pedro Ascencio Alquisiras & Oaxaca & Santa Cruz Acatepec \\
\hline Guerrero & San Miguel Totolapan & Oaxaca & Santa Cruz Zenzontepec \\
\hline Guerrero & Tlacoachistlahuaca & Oaxaca & Santa Inés del Monte \\
\hline Guerrero & Tlacoapa & Oaxaca & Santa Lucía Miahuatlán \\
\hline Guerrero & Xalpatláhuac & Oaxaca & Santa Lucía Monteverde \\
\hline Guerrero & Zapotitlán Tablas & Oaxaca & Santa María la Asunción \\
\hline Guerrero & Acatepec & Oaxaca & Santa María Chilchotla \\
\hline Guerrero & Cochoapa el Grande & Oaxaca & Santa María Peñoles \\
\hline Guerrero & José Joaquín de Herrera & Oaxaca & Santa María Temaxcaltepec \\
\hline Guerrero & Iliatenco & Oaxaca & Santiago Amoltepec \\
\hline Nayarit & Del Nayar & Oaxaca & Santiago Ixtayutla \\
\hline
\end{tabular}

Fuente: elaboración propia con información del Inegi (2011). 
Continuación...

\begin{tabular}{clcl}
\hline Entidad & \multicolumn{1}{c}{ Municipio } & Entidad & \multicolumn{1}{c}{ Municipio } \\
\hline Oaxaca & Santiago Tlazoyaltepec & Veracruz & Atlahuilco \\
& Santo Domingo de Morelos & Mixtla de Altamirano \\
& Santo Domingo Tepuxtepec & Los Reyes \\
& Santos Reyes Yucuná & Soledad Atzompa \\
Puebla & Coyomeapan & Tehuipango \\
& Eloxochitlán & Tequila \\
& Zoquitlán & Texcatepec \\
Veracruz & Astacinga & Zontecomatlán de López y Fuentes \\
\hline
\end{tabular}

Fuente: elaboración propia con datos del Inegi (2011).

Cuadro 8

Municipios del Grupo B

\begin{tabular}{clll}
\hline Entidad & \multicolumn{1}{c}{ Municipio } & Entidad & \multicolumn{1}{c}{ Municipio } \\
\hline Chiapas & Francisco León & Oaxaca & San Simón Zahuatlán \\
& Ocotepec & & Santa María Apazco \\
& Pantepec & Santa María Tepantlali \\
& Tumbalá & & Santa María Tlalixtac \\
& San Andrés Duraznal & Santiago Texcalcingo \\
Guerrero & Ahuacuotzingo & Santiago Xanica \\
& General Heliodoro Castillo & & Santiago Yaitepec \\
& Xochistlahuaca & Yogana \\
& Zitlala & Puebla & Camocuautla \\
Oaxaca & San Bartolomé Ayautla & & Chiconcuautla \\
& San Francisco Chapulapa & Huehuetla \\
& San Francisco Huehuetlán & & Hueytlalpan \\
& San Francisco Tlapancingo & San Felipe Tepatlán \\
& San José Independencia & & San Sebastián Tlacotepec \\
& San Juan Juquila Mixes & Veracruz & Filomeno Mata \\
& San Lorenzo Cuaunecuiltitla & & Ilamatlán \\
& San Miguel Ahuehuetitlán & Mecatlán \\
San Miguel Santa Flor & Soteapan \\
San Pedro el Alto & Tlaquilpa \\
San Pedro Ocopetatillo & & Xoxocotla \\
San Pedro y San Pablo Ayutla & & \\
\hline
\end{tabular}

Fuente: elaboración propia con información de Inegi (2011). 


\section{Cuadro 9 \\ Municipios del Grupo C}

\begin{tabular}{clll}
\hline Entidad & \multicolumn{1}{c}{ Municipio } & Entidad & \multicolumn{1}{c}{ Municipio } \\
\hline Chiapas & Altamirano & Oaxaca & San Mateo Piñas \\
& Oxchuc & San Miguel Huautla \\
Chihuahua & Batopilas & San Miguel Piedras \\
& Guachochi & San Pablo Tijaltepec \\
& Guadalupe y Calvo & San Pedro Mártir \\
& Morelos & San Sebastián Río Hondo \\
Guerrero & Uruachi & Coahuayutla de José María & Santa Catarina Loxicha \\
& Izazaga & Santa Catarina Yosonotú \\
Jalisco & San Luis Acatlán & Mezquitic & \\
& Asunción Ocotlán & Santa Cruz Itundujia \\
& Coatecas Altas & Santa Cruz Tacahua \\
& Magdalena Mixtepec & Santa María Ozolotepec \\
& San Cristóbal Amoltepec & Santa María Yolotepec \\
& San Esteban Atatlahuca & Santiago Apoala \\
& San Francisco Cahuacuá & Santiago Nundiche \\
& San Francisco Logueche & Santiago Zacatepec \\
& San Juan Lachigalla & Santo Domingo Nuxá \\
& San Juan Tamazola & Santo Luis P. & Aquismón \\
& San Martín Peras & Santa Catarina \\
& San Mateo del Mar & \\
\hline
\end{tabular}

Fuente: elaboración propia con información de Inegi (2011).

\section{Fuentes consultadas}

Chakravarty, Satya (2003), "A generalized human development index”, Review of Development Economics, 7 (1), Blackwell, Oxford, pp. 99-114.

Conapo (Consejo Nacional de Población) (2011), “Índice de marginación por entidad y municipio 2010”, Conapo, México.

Conapo (Consejo Nacional de Población) (2001), "Índices de Desarrollo Humano 2000", Conapo, México.

Coneval (Consejo Nacional de Evaluación de la Política de Desarrollo Social) (2013a), "Medición de la pobreza", Coneval, <http://www. 
coneval.org.mx/Medicion/MP/Paginas/Que-es-la-medicionmultidimensional-de-la-pobreza.aspx>, 29 de agosto de 2013.

Coneval (Consejo Nacional de Evaluación de la Política de Desarrollo Social) (2013b), "Evolución de las dimensiones de la pobreza 1990-2012", <http://www.coneval.org.mx/Medicion/Paginas/ Evolucion-de-las-dimensiones-de-la-pobreza-1990-2010-.aspx>, 2 de septiembre de 2013.

Coneval (Consejo Nacional de Evaluación de la Política de Desarrollo Social) (2012), "Evaluación de impacto de la Estrategia 100x100", <http://www.coneval.org.mx/Informes/Evaluacion/Impacto/ Evaluacion_de_impacto_de_la_Estrategia_100x100.pdf $>, 28 \mathrm{de}$ agosto de 2013.

Coneval (Consejo Nacional de Evaluación de la Política de Desarrollo Social) (2011a), Comunicado de prensa No. 007, <http://www. coneval.org.mx/SalaPrensa/Documents/Comunicado007_Reconocimiento_Buenas_Practicas\%20(1).pdf>, 28 de agosto de 2013.

Coneval (Consejo Nacional de Evaluación de la Política de Desarrollo Social) (2011b), "Índice de Rezago Social 2010 a nivel municipal y por localidad", <http://www.coneval.org.mx/Medicion/IRS/ Paginas/\%C3\%8Dndice-de-Rezago-social-2010.aspx>, 28 de agosto de 2015.

Coneval (Consejo Nacional de Evaluación de la Política de Desarrollo Social) (2007), "Los mapas de la pobreza en México", <http:// siic.ucol.mx/Archivos_prov\%5CLos_mapas_de_la_pobreza_en_ Mexico_Presentacion_en_PP.pdf>, 5 de septiembre de 2013.

Cortés, Fernando, Daniel Hernández, Enrique Hernández, Miguel Székely y Hadid Vera (2003), "Evolución y características de la pobreza en México en la última década del siglo XX", Economía Mexicana, 7 (2), CIDE, México, pp. 295-325.

De Maesschalck, Roy, Delphine Jouan-Rimbaud y Désiré L. Massart (2000), "The mahalanobis distance", Chemometrics and intelligent laboratory systems, 50 (1), Elsevier, Exeter, pp. 1-18.

Foa, Roberto y Jeffery Tanner (2012), "Methodology of the Indices of Social Development”, ISD working paper series, 2012-04, pp. 1-66, $<$ http://hdl.handle.net/1765/50510>, 28 de agosto de 2015. 
Hagerty, Michael, Robert C. Cummins, Abbott L. Ferris, Kenneth Land, Alex C. Michalos, Mark Peterson, Andrew Sharpe, Joshep Sirgy y Joachim Vogel (2001), "Quality of life indexes for national policy: review and agenda for research", Social Indicators Research, 50 (1), Kluwer Academic, Amsterdam, pp. 1-96.

Herrero, Carmen, Ricardo Martínez y Antonio Villar (2010), "Multidimensional social evaluation: a application to the measurement of human development", Review of Income and Wealth, 56 (3), Blackwell, Oxford, pp. 483-497.

Inegi (Instituto Nacional de Estadística y Geografía) (2011), “Censo de Población y Vivienda 2010. Principales resultados por localidad”, Inegi, Aguascalientes.

Johnson, Richard y Dean Wichern (1999), Applied multivariate statistical analysis, Prentice-Hall, Nueva Jersey.

Klugman, Jeni, Francisco Rodríguez y Hyung-Jin Choi (2011), "The hdi 2010: new controversies, old critiques", The Journal of Economic Inequality, 9 (2), Springer, Nueva York, pp. 249-288.

Mahalanobis, Prasanta Chandra (1936), "On the generalized distance in statistics", Proceedings of the National Institute of Sciences, núm. 2, Calcuta, pp. 49-55.

McGillivray, Mark (1991), “The Human Development Index: Yet another Redundant Composite Development Indicator?", World Development, 19 (10), Pergamon Press, Oxford, pp. 1461-1468.

McLachlan, Goeffrey J. (2000), "Mahalanobis distance", Chemometrics and Intelligent Laboratory Systems, 50 (1), Elsevier, pp. 1-18.

Michinaka, Maki (2008), "A ranking of Human Development without Aggregation: An Alternative to the HDI", Centre of Excellencel Research on Economics Systems Discussion Paper Series, No. 233, Hitotsubashi University, Tokyo.

Mishra, RK (2007), "A comparative study of various inclusive indices and the index constructed by the principal component analysis", Munich Personal RePEc Archive Paper No. 3377, http://mpra. ub.uni-muenchen.de/3377/, 3 de octubre de 2013. 
Nardo, Michela, Michaela Saisana, Andrea Saltelli, Stefano Tarantola, Anders Hoffman, Enrico Giovannini (2005), "Handbook on Constructing Composite Indicators: Methodology and User Guide", OECD Statistics Working Papers, No. 2005/03, OECD Publishing, París.

Noorbakhsh, Farhad (1998), "A Modified Human Development Index", World Development, 26 (3), Elsevier, Exeter, pp. 517-528.

Osberg, Lars y Andrew Sharpe (2005), "How should we measure the 'economic' aspects of well-being?", Review of Income and Wealth, 51 (2), Blackwell, Oxford, pp. 311-336.

PNUD (Programa de las Naciones Unidas para el Desarrollo) (2015), "Índice de Desarrollo Humano para las entidades federativas México 2015”, PNUD, México.

PNUD (Programa de las Naciones Unidas para el Desarrollo) (2014), "Índice de Desarrollo Humano Municipal en México: Nueva Metodología”, PNUD, México.

PNUD (Programa de las Naciones Unidas para el Desarrollo) (2010), Human Development Report 2010. The Real Wealth of Nations: Pathways to Human Development, Palgrave Macmillan, Nueva York. Sedesol (Secretaría de Desarrollo Social) (2010), "Estrategia 100x100", <http://www.estrategia100x100.gob.mx>, 28 de agosto de 2013.

PNUD (Programa de las Naciones Unidas para el Desarrollo) (1990), Human Development Report 1990. Concept and Measurement of Human Development, Oxford University Press, Nueva York.

Székely, Miguel, Luis López-Calva, Álvaro Meléndez, Ericka Rascón, Lourdes Rodríguez-Chamussy (2007), "Poniendo a la pobreza de ingresos y a la desigualdad en el mapa de México", Economía Mexicana, 16 (2), CIDE, México, pp. 239-303.

Sen, Amartya (1985), Commodities and capabilities, North-Holland, Amsterdam.

Zambrano, Eduardo (2011), "An axiomatization of the human development index", Human Development Research Report, 2011/10, PNUD, Nueva York. 
Recibido: 8 de noviembre de 2014. Corregido: 9 de octubre de 2015. Aceptado: 22 de noviembre de 2015.

Francisco José Zamudio-Sánchez. Mexicano. Ph. D. con mayor en estadística, Universidad Estatal de Iowa. Profesor-investigador de tiempo completo, Universidad Autónoma Chapingo, Departamento de Estadística, Matemática y Cómputo. Miembro del Sistema Nacional de Investigadores nivel II; sus líneas de investigación: estadística social y análisis multivariado. Entre sus publicaciones recientes se encuentran, en coautoría: "Dinámica de la calidad e inequidad del desarrollo humano en la región noreste de México, 1995-2005”, Estudios fronterizos, 14 (27), Universidad Autónoma de Baja California, México, pp. 183-216 (2013); "Análisis sobre la evolución del desarrollo humano en la Península de Yucatán”, Economía, Sociedady Territorio, XII (40), El Colegio Mexiquense, A. C., Zinacantepec, pp. 543-596 (2012); "Una estrategia para portafolios con fondos cotizados", Revista Española de Financiación y Contabilidad, XLI (155), Asociación Española de Contabilidad y Administración de Empresas, Madrid, pp. 417-444 (2012).

Roxana Ivette Arana-Ovalle. Mexicana. Maestra en Ciencias en Estadística, Colegio de Posgraduados, México. Investigadora asociada Universidad Autónoma Chapingo, Departamento de Estadística, Matemática y Cómputo. Líneas de investigación: estadística social, análisis multivariado. Publicaciones recientes, en coautoría: "Una estrategia para portafolios con fondos cotizados", Revista Española de Financiación y Contabilidad, XLI (155), Asociación Española de Contabilidad y Administración de Empresas, Madrid, pp. 417-444 (2012); Estudio sobre la violencia de género: la otra cara de la Universidad Autónoma Chapingo, Universidad Autónoma Chapingo, México (2013).

Javier Santibáńez-Cortés. Mexicano. Licenciado en Estadística, Universidad Autónoma Chapingo, México. Asistente de Investigador, Universidad Autónoma Chapingo, Departamento de Estadística, Matemática y Cómputo, Líneas de investigación: estadística social, análisis multivariado. 